\title{
On the Theory of Pfaffian Orientations. I. Perfect Matchings and Permanents.
}

\author{
*Anna Galluccio \\ Istituto di Analisi dei Sistemi ed \\ Informatica - CNR \\ viale Manzoni 30 \\ 00185 Roma \\ ITALY \\ galluccio@iasi.rm.cnr.it
}

\author{
${ }^{\dagger}$ Martin Loebl \\ Department of Applied Mathematics \\ Charles University \\ Malostranske n. 25 \\ 11800 Praha 1 \\ CZECH REPUBLIC \\ loebl@kam.ms.mff.cuni.cz
}

Received May 7, 1998; Accepted October 28, 1998.

\begin{abstract}
Kasteleyn stated that the generating function of the perfect matchings of a graph of genus $g$ may be written as a linear combination of $4^{g}$ Pfaffians. Here we prove this statement. As a consequence we present a combinatorial way to compute the permanent of a square matrix.
\end{abstract}

Mathematical Reviews Subject Numbers 05B35, 05C15, 05A15 


\section{Introduction}

The theory of Pfaffian orientations of graphs has been introduced by Kasteleyn [7, 6, 5] in early sixties to solve some enumeration problems arising from statistical physics $[4,10]$. He proved fundamental results in the planar case and extended his approach to toroidal grids $[5,6,7]$. The case of general toroidal graphs was also considered in an unpublished manuscript by Barahona [1].

In the present paper we extend the method proposed by Kasteleyn and we prove that the generating function of the perfect matchings of a graph of genus $g$ may be obtained as a linear combination of $4^{g}$ Pfaffians. As a consequence, we provide a new technique to compute permanents of square matrices, which completes the scheme proposed by Pólya in [9].

A graph is a pair $G=(V, E)$ where $V$ is a set of vertices and $E$ is a set of unordered pairs of elements of $V$, called edges. In this paper we shall consider only graphs with finite number of vertices. If $e=x y$ is an edge then the vertices $x, y$ are called endvertices of $e$. We associate with each edge $e$ of $G$ a variable $x_{e}$ and we let $x=\left(x_{e}: e \in E\right)$. For each $M \subset E$, let $x(M)$ denote the product of the variables of the edges of $M$.

A graph $G^{\prime}=\left(V^{\prime}, E^{\prime}\right)$ is called a subgraph of a graph $G=(V, E)$ if $V^{\prime} \subset V$ and $E^{\prime} \subset E$. A perfect matching of a graph is a set of disjoint edges, whose union equals the set of the vertices.

Let $\left\{v_{1}, e_{1}, v_{2}, e_{2}, \ldots, v_{i}, e_{i}, v_{i+1}, \ldots, e_{n}, v_{n+1}\right\}$ be a sequence such that each $v_{j}$ is a vertex of a graph $G$, each $e_{j}$ is an edge of $G$ and $e_{j}=v_{j} v_{j+1}$, and $v_{i} \neq v_{j}$ for $i<j$ except if $i=1$ and $j=n+1$. If also $v_{1} \neq v_{n+1}$ then $P$ is called a path of $G$. If $v_{1}=v_{n+1}$ then $P$ is called a cycle of $G$. In both cases the length of $P$ equals $n$. When no confusion arises we shall also denote paths by simply listing their edges, namely $P=\left(e_{1}, e_{2}, \ldots, e_{n}\right)$.

A graph $G=(V, E)$ is connected if it has a path between any pair of vertices, and it is 2-connected if the graph $G_{v}=(V-\{v\},\{e \in E ; v \notin e\})$ is connected for each vertex $v$ of $G$. Each maximal 2-connected subgraph of $G$ is called a 2-connected component of $G$.

Let $A \Delta B$ denote the symmetric difference of the sets $A$ and $B$ and let $a \stackrel{2}{=} b$ denote $a=b$ modulo 2 .

Let $M, N$ be two perfect matchings of a graph $G$. Then $M \Delta N$ consists of vertex disjoint cycles of even length. These cycles are called alternating cycles of $M$ and $N$.

An orientation of a graph $G=(V, E)$ is a digraph $D=(V, A)$ obtained from $G$ by fixing an orientation of each edge of $G$, i.e., by ordering the elements of each edge of $G$. The elements of $A$ are called arcs.

Let $C$ be a cycle of $G$ and let $D$ be an orientation of $G$. $C$ is said to be clockwise even in $D$ if it has an even number of edges directed in $D$ in agreement with the clockwise traversal. Otherwise $C$ is called clockwise odd.

Definition 1.1 The generating function of the perfect matchings of $G$ is the polynomial $\mathcal{P}(G, x)$ which equals the sum of $x(P)$ over all perfect matchings $P$ of $G$. 
Definition 1.2 Let $G$ be a graph and let $D$ be an orientation of $G$. Let $M$ be a perfect matching of $G$. For each perfect matching $P$ of $G$ let $\operatorname{sgn}(D, M \Delta P)=(-1)^{n}$ where $n$ is the number of clockwise even alternating cycles of $M$ and $P$, and let $\mathcal{P}(D, M)$ be the sum of $\operatorname{sgn}(D, M \Delta P) x(P)$ over all perfect matchings $P$ of $G$.

Definition 1.3 Let $G=(V, E)$ be a graph with $2 n$ vertices and $D$ an orientation of $G$. Denote by $A(D)$ the skew-symmetric matrix with the rows and the columns indexed by $V$, where $a_{v w}=x_{v w}$ in case $(v, w)$ is an arc of $D, a_{v w}=-x_{v w}$ in case $(w, v)$ is an arc of $D$, and $a_{v w}=0$ otherwise.

The Pfaffian of the skew-symmetric matrix $A(D)$ is defined as

$$
P f(A(D))=\sum_{P} s^{*}(P) a_{i_{1} j_{1}} \cdots a_{i_{n} j_{n}}
$$

where $P=\left\{\left\{i_{1} j_{1}\right\}, \cdots,\left\{i_{n} j_{n}\right\}\right\}$ is a partition of the set $\{1, \ldots, 2 n\}$ into pairs, $i_{k}<$ $j_{k}$ for $k=1, \ldots, n$, and $s^{*}(P)$ equals the sign of the permutation $i_{1} j_{1} \ldots i_{n} j_{n}$ of $12 \ldots(2 n)$.

Each nonzero term of the expansion of the Pfaffian of $A(D)$ equals $x(P)$ or $-x(P)$ where $P$ is a perfect matching of $G$. If $s(D, P)$ denote the sign of the term $x(P)$, we have that

$$
P f(A(D))=\sum_{P} s(D, P) x(P)
$$

The following theorem was proved by Kasteleyn [5].

Theorem 1.4 Let $G$ be a graph and $D$ an orientation of $G$. Let $P, M$ be two perfect matchings of $G$. Then

$$
s(D, P)=s(D, M) \operatorname{sgn}(D, M \Delta P) .
$$

Hence,

$P f(A(D))=\sum_{P} s(D, P) x(P)=s(D, M) \sum_{P} \operatorname{sgn}(D, M \Delta P) x(P)=s(D, M) \mathcal{P}(D, M)$.

The relevance of Pfaffians in our context lies in the fact that, despite their similarity with the permanent, they are polynomial time computable for skew-symmetric matrices (see [2]). In fact, see [7] for a proof.

Theorem 1.5 Let $G$ be a graph and let $D$ be an orientation of $G$. Then

$$
P f^{2}(A(D))=\operatorname{det}(A(D)) \text {. }
$$

In [5] Kasteleyn introduced the following notion: 
Definition 1.6 A graph $G$ is called Pfaffian if it has a Pfaffian orientation, i.e., an orientation such that all alternating cycles with respect to an arbitrary fixed perfect matching $M$ of $G$ are clockwise odd.

Hence if a graph $G$ has a Pfaffian orientation $D$ then the signs $s(D, P)$ are equal for all perfect matchings $P$ of $G$ and $\mathcal{P}(G, x)^{2}=P f^{2}(A(D))=\operatorname{det}(A(D))$.

An embedding of a graph on a surface is defined in a natural way: the vertices are embedded as points, and each edge is embedded as a continuous non-self-intersecting curve connecting the embeddings of its endvertices. The interiors of the embeddings of the edges are pairwise disjoint and the interiors of the curves embedding edges do not contain points embedding vertices.

A graph is called planar if it may be embedded on the plane. A plane graph is a planar graph together with its planar embedding. The embedding of a plane graph partitions the plane into connected regions called faces. The (unique) unbounded face is called outer face and the bounded faces are called inner faces.

Let $G$ be a plane graph. A subgraph of $G$ consisting of the vertices and the edges embedded on the boundary of a face will also be called a face. If a plane graph is 2 -connected then each face is a cycle.

Kasteleyn [5] observed that the planar graphs have a Pfaffian orientation; more specifically, he proved that

Theorem 1.7 Every plane graph has a Pfaffian orientation such that all inner faces are clockwise odd.

Proof. Let $G$ be a plane graph, and let $M$ be its perfect matching. Each alternating cycle of $M$ belongs to a 2-connected component of $G$.

Observe that $G$ has an orientation so that each inner face of each 2-connected component of $G$ is clockwise odd. Each such face 'encircles' no vertex of the corresponding 2-connected component. Let $W$ be a 2-connected component of $G$. Observe that the orientation we constructed has the property that a cycle $C$ of $W$ is clockwise odd if and only if $C$ encircles an even number of vertices of $W$. Let $C$ be an alternating cycle of $M$ and let $W$ be a 2-connected component of $G$ which contains $C$. Then $C$ encircles an even number of vertices of $W$ and hence it is clockwise odd.

\section{Embeddings and Pfaffian orientations}

The genus $g$ of a graph $G$ is that of the orientable surface $\mathcal{S} \subset \mathbb{R}^{3}$ of minimal genus on which $G$ may be embedded. Any orientable surface of genus $g$ has a polygonal representation obtained by cutting the $g$ handles of its space model. In what follows we base our working definition of a surface on this concept.

Definition $2.1 A$ surface $S_{g}$ of genus $g$ consists of a base $B_{0}$ and $2 g$ bridges $B_{j}^{i}$, $i=1, \ldots, g$ and $j=1,2$, where 
i) $B_{0}$ is a convex $4 g$-gon with vertices $a_{1}, \ldots, a_{4 g}$ numbered clockwise;

ii) $B_{1}^{i}, i=1, \ldots, g$, is a 4-gon with vertices $x_{1}^{i}, x_{2}^{i}, x_{3}^{i}, x_{4}^{i}$ numbered clockwise. It is glued with $B_{0}$ so that the edge $\left[x_{1}^{i}, x_{2}^{i}\right]$ of $B_{1}^{i}$ is identified with the edge $\left[a_{4(i-1)+1}, a_{4(i-1)+2}\right]$ of $B_{0}$ and the edge $\left[x_{3}^{i}, x_{4}^{i}\right]$ of $B_{1}^{i}$ is identified with the edge $\left[a_{4(i-1)+3}, a_{4(i-1)+4}\right]$ of $B_{0}$;

iii) $B_{2}^{i}, i=1, \ldots, g$, is a 4-gon with vertices $y_{1}^{i}, y_{2}^{i}, y_{3}^{i}, y_{4}^{i}$ numbered clockwise. It is glued with $B_{0}$ so that the edge $\left[y_{1}^{i}, y_{2}^{i}\right]$ of $B_{2}^{i}$ is identified with the edge $\left[a_{4(i-1)+2}, a_{4(i-1)+3}\right]$ of $B_{0}$ and the edge $\left[y_{3}^{i}, y_{4}^{i}\right]$ of $B_{2}^{i}$ is identified with the edge $\left[a_{4(i-1)+4}, a_{4(i-1)+5(\bmod 4 g)}\right]$ of $B_{0}$.

Observe that in Definition 2.1 we denote by $[a, b]$ edges of polygons and not edges of graphs. The usual representation in the space of an orientable surface $\mathcal{S}$ of genus $g$ may be then obtained from its polygonal representation $S_{g}$ by the following operation: for each bridge $B$, glue together the two segments which $B$ shares with the boundary of $B_{0}$, and delete $B$.

Definition 2.2 A graph $G$ is called a g-graph if it may be embedded on $S_{g}$ so that all the vertices belong to the base $B_{0}$, and the embedding of each edge uses at most one bridge. The set of the edges embedded entirely on the base will be denoted by $E_{0}$ and the set of the edges embedded on the bridge $B_{j}^{i}$ will be denoted by $E_{j}^{i}, i=1, \ldots, g$, $j=1,2$. If a g-graph $G$ satisfies the following further conditions:

1. the outer face of $G_{0}=\left(V, E_{0}\right)$ is a cycle, and it is embedded on the boundary of $B_{0}$,

2. if $e \in E_{1}^{i}$ then $e$ is embedded entirely on $B_{1}^{i}$ and one endvertex of e belongs to $\left[x_{1}^{i}, x_{2}^{i}\right]$ and the other one belongs to $\left[x_{3}^{i}, x_{4}^{i}\right]$. Similarly, if $e \in E_{2}^{i}$ then $e$ is embedded entirely on $B_{2}^{i}$ and one endvertex of e belongs to $\left[y_{1}^{i}, y_{2}^{i}\right]$ and the other one belongs to $\left[y_{3}^{i}, y_{4}^{i}\right]$.

3. each vertex is incident with at most one edge which does not belong to $E_{0}$,

4. $G_{0}$ has a perfect matching,

then we say that $G$ is a proper g-graph.

Given a proper $g$-graph $G$, we denote by $C_{0}$ the cycle which forms the outer face of $E_{0}$; then, we fix a perfect matching of $G_{0}$ and denote it by $M_{0}$.

Definition 2.3 Let $G$ be a proper g-graph and let $G_{j}^{i}=\left(V, E_{0} \cup E_{j}^{i}\right)$. If we draw $B_{0} \cup B_{j}^{i}$ on the plane as follows: $B_{0}$ is unchanged, and the edge $\left[x_{1}^{i}, x_{4}^{i}\right] \quad\left(\left[y_{1}^{i}, y_{4}^{i}\right]\right.$ respectively) of $B_{j}^{i}$ is drawn so that it belongs to the external boundary of $B_{0} \cup B_{j}^{i}$, we obtain a planar embedding of $G_{j}^{i}$. This embedding will be called planar projection of $E_{j}^{i}$ outside $B_{0}$. 
Definition 2.4 Let $G=(V, E)$ be a proper g-graph. A Pfaffian orientation $D_{0}$ of $G_{0}$ such that each inner face of each 2-connected component of $G_{0}$ is clockwise odd in $D_{0}$ is called a basic orientation of $G_{0}$.

Note that a basic orientation always exists for a planar graph by Theorem 1.7.

Definition 2.5 Let $G=(V, E)$ be a proper g-graph and $D_{0}$ a basic orientation of $G_{0}$. We define the orientation $D_{j}^{i}$ of each $G_{j}^{i}$ as follows: We consider $G_{j}^{i}$ embedded on the plane by the planar projection of $E_{j}^{i}$ outside $B_{0}$ (see Definition 2.3), and complete the basic orientation $D_{0}$ of $G_{0}$ to an orientation of $G_{j}^{i}$ so that each inner face of each 2 -connected component of $G_{j}^{i}$ is clockwise odd.

The orientation $-D_{j}^{i}$ is defined by reversing the orientation $D_{j}^{i}$ of $G_{j}^{i}$.

Observe that after fixing a basic orientation $D_{0}$, the orientation $D_{j}^{i}$ is uniquely determined for each $i, j$.

Definition 2.6 Let $G$ be a proper g-graph, $g \geq 1$. An orientation $D$ of $G$ which equals the basic orientation $D_{0}$ on $G_{0}$ and which equals $D_{j}^{i}$ or $-D_{j}^{i}$ on $E_{j}^{i}$ is called relevant. We define its type $r(D) \in\{+1,-1\}^{2 g}$ as follows: For $i=0, \ldots, g-1$ and $j=1,2, r(D)_{2 i+j}$ equals +1 or -1 according to the sign of $D_{j}^{i+1}$ in $D$.

Definition 2.7 Let $G$ be a proper $g$-graph and let $A$ be a subset of its edges. The type of $A$ is a vector $t(A) \in\{0,1\}^{2 g}$ defined as follows: For $i=0, \ldots, g-1$ and $j=1,2$, we let $t(A)_{2 i+j}$ equals the number of edges of $A$ which belong to $E_{j}^{i+1}$, modulo 2 .

Let $C R(A) \stackrel{2}{=} \sum_{i=0}^{g-1} t(A)_{2 i+1} \cdot t(A)_{2 i+2}$ denote the number of crossings of the embeddings of the edges of $A$, after making planar projections of $E_{j}^{i}$ for all $i, j$.

Let $B R(A)$ denote the subset of edges of $A$ which do not belong to $E_{0}$. For each $e \in B R(A)$, let $d(e)=2 i+j$ if $e \in E_{j}^{i+1}$.

We complete the section with a lemma.

Lemma 2.8 Let $G$ be a proper g-graph. Let $C_{1}, \ldots, C_{k}$ be vertex-disjoint cycles of $G$ and let $\mathcal{C}$ denote their union. Then

$$
C R(\mathcal{C}) \stackrel{2}{=} \sum_{i=1}^{k} C R\left(C_{i}\right)
$$

Proof. Let us embed the cycles $C_{1}, \ldots, C_{k}$ using the planar projections of $E_{j}^{i}$ outside $B_{0}$ by Definition 2.7. Then $C R(\mathcal{C})$ equals the total number of crossings of $\mathcal{C}$ (modulo 2 ). Now, each cycle $C_{l}, l=1, \ldots, k$ is represented as a closed curve in the plane and each pair of cycles $C_{i}$ and $C_{j}, i \neq j$, intersects an even number of times. Hence the sum (modulo 2) of the number of crossings between pairs of cycles $C_{i}$ and $C_{j}, i \neq j$, is 0 and does not affect $C R(\mathcal{C})$. Each of the remaining crossings is a crossing of some $C_{l}, l=1, \ldots, k$, with itself and the lemma follows. 


\section{Perfect matchings}

Through this section, the graph $G$ will be a proper $g$-graph embedded on a fixed surface $S_{g}$. We also fix a perfect matching $M_{0}$ of $G_{0}$.

The aim of this section is to prove that, for any perfect matching $P$, the $\operatorname{sgn}\left(D, M_{0} \Delta P\right)$ depends only on the vectors $t\left(M_{0} \Delta P\right)$ and $r(D)$.

Given an orientation $D$ of $G$ and an even length cycle $C$ of $G$, we denote by $l_{D}(C)$ the number of arcs of $C$ directed in agreement with any of the two possible ways of traversing $C$, modulo 2. For short, any alternating cycle with respect to $M_{0}$ will be simply called an alternating cycle. In order to prove our statement, we consider first the case that $M_{0} \Delta P$ consists of exactly one alternating cycle.

Theorem 3.1 Let $G$ be a proper g-graph and let $D$ be a relevant orientation of $G$. If $C$ is an alternating cycle of $G$, then

$$
l_{D}(C) \stackrel{2}{=}|B R(C)|-1-C R(C)+\frac{1}{2} \sum_{e \in B R(C)}\left(r(D)_{d(e)}+1\right)
$$

Proof. We assume without loss of generality that $G=C \cup C_{0} \cup M_{0}$, where $C_{0}$ is the outer face of $G_{0}$ and $M_{0}$ is the fixed perfect matching of $G_{0}$. Let $D_{0}$ be the basic orientation of $G_{0}$.

Claim 1. If $C$ intersects at most one of $E_{1}^{i}, E_{2}^{i}$, for each $i=1, \ldots, g$, then

$$
l_{D}(C) \stackrel{2}{=}|B R(C)|-1+\frac{1}{2} \sum_{e \in B R(C)}\left(r(D)_{d(e)}+1\right) .
$$

A cycle $C$ satisfying the properties of Claim 1 may be embedded without crossings using the planar projection of each $E_{j}^{i}$ outside $B_{0}$. Hence $l_{D}(C)=1$ if and only if $\left|\left\{e \in B R(C): r(D)_{d(e)}=-1\right\}\right| \stackrel{2}{=} 0$.

End of Claim 1.

The proof is by induction on $|B R(C)|$. The case $|B R(C)|=0$ is proved by Claim 1. By induction we assume that

$$
l_{W}\left(C^{\prime}\right) \stackrel{2}{=}\left|B R\left(C^{\prime}\right)\right|-1-C R\left(C^{\prime}\right)+\frac{1}{2} \sum_{e \in B R\left(C^{\prime}\right)}\left(r(W)_{d(e)}+1\right)
$$

for any alternating cycle $C^{\prime}$ of a proper $g$-graph $H$, with relevant orientation $W$, such that $\left|B R\left(C^{\prime}\right)\right|<|B R(C)|$.

We distinguish two cases.

Case 1. There exists a bridge $B=B_{j}^{i}$ containing more than one edge of $C$.

Let $e=u_{1} u_{2}$ and $f=v_{1} v_{2}$ be two edges of $C \cap E_{j}^{i}$ which see each other on $B$, i.e., there is no other edge of $C$ drawn between them on $B$. Without loss of generality, let $e$ be nearer to the edge $\left[a_{2(i-1)+j}, a_{2(i-1)+j+3}\right]$ of $B=B_{j}^{i}$ than $f$ and let $u_{1}, v_{1}$ and $u_{2}, v_{2}$ belong to the edge $\left[a_{2(i-1)+j}, a_{2(i-1)+j+1}\right]$ and $\left[a_{2(i-1)+j+2}, a_{2(i-1)+j+3}\right]$, respectively. Since $e, f$ do not belong to $E_{0}$, they are not edges of $M_{0} \subset E_{0}$. 
Let $R_{i}$ be the subpath of $C_{0}$ from $u_{i}$ to $v_{i}, i=1,2$, and let $R$ be the cycle of $G$ consisting of $\left(R_{1}, f, R_{2}, e\right)$. By the choice of $e, f$, the cycle $R$ is the boundary of a face of the planar projection of $G_{j}^{i}=\left(V, E_{0} \cup E_{j}^{i}\right)$ outside $B_{0}$. Observe that $l_{W}(R)=1$ for each relevant orientation $W$ of $G$, since $R$ contains two edges embedded outside $B_{0}$.

Let us introduce a new edge $h$ (not belonging to $G$ ), between the endvertices of $e, f$ such that one of two cycles $\bar{H}_{1}, \bar{H}_{2}$ formed by $h$ and $C$ and containing $h$ is alternating. Without loss of generality, let $h$ have $u_{1}$ as an endvertex. Hence we have that $h=u_{1} v_{1}$ or $h=u_{1} v_{2}$.

We may assume without loss of generality that $\bar{H}_{2}$ is alternating. Hence $\bar{H}_{1}$ contains both $e, f$. Note that $\bar{H}_{1}$ consists of an even number of edges. We denote by $h_{1}, h_{2}$ the two arcs with the same endvertices as $h$, directed oppositely. Let $D^{\prime}=D \cup\left\{h_{1}, h_{2}\right\}$. Let $H_{i}$ be the subdigraph of $D^{\prime}$ which is the orientation of $\bar{H}_{i}$ using $h_{i}, i=1,2$. Observe that $l_{D}(C)=l_{D^{\prime}}\left(H_{1}\right)+l_{D^{\prime}}\left(H_{2}\right)$.

Subcase 1.1: $h_{1}=u_{1} v_{1}$.

We adjust the boundary of $B_{0}$ by replacing $\left\{R_{1}\right\}$ with $h_{1}, h_{2}$. Observe that $C R(C) \stackrel{2}{=} C R\left(H_{1}\right)+C R\left(H_{2}\right)$ : attention should be drawn to the question of how crossings of $C$ with itself are manifested as crossings of $H_{1}$ or $H_{2}$, when all $E_{j}^{i}$ are projected outside of $B_{0}$ (see Definition 2.3). If two edges of $C$ cross and they are not separated in $C$ by the endvertices of $h_{1}$, then that crossing counts as a crossing with in $H_{1}$ or $H_{2}$. We must therefore consider the parity of the number of crossings of $C$ where the crossed edges are separated in $C$ by the endvertices of $h_{1}$. These crossings are counted as crossings of $H_{1}$ with $H_{2}$. If the number of such crossings of $C$ is odd, then there must be an additional crossing of $H_{1}$ with $H_{2}$, since the total number of crossings of $H_{1}$ with $H_{2}$ must be even. Since $h_{1}$ and $h_{2}$ do not cross, this additional crossing must occur at an endvertex of $h_{1}$. It is easy to see that in the present case there is no such crossing, and so, there are an even number of crossings of $C$ where the crossed edges are separated in $C$ by the ends of $h$. The required congruence therefore follows in this case.

We construct now two digraphs $D_{1}, D_{2}$ as follows:

- $D_{1}$ is obtained from $D-\{e, f\}$ by adding new vertices $u_{1}^{\prime}, v_{1}^{\prime}$ of degree 2 , incident with new $\operatorname{arcs} e^{\prime}, f^{\prime}, h_{1}^{\prime}$. The $\operatorname{arcs} e^{\prime}, f^{\prime}, h_{1}^{\prime}$ are obtained from $e, f, h_{1}$ by replacing $u_{1}$ by $u_{1}^{\prime}$ and $v_{1}$ by $v_{1}^{\prime}$. We adjust the boundary of $B_{0}$ by replacing $\left\{R_{2}\right\}$ with $\left\{e^{\prime}, f^{\prime}, h_{1}^{\prime}\right\}$. Finally we add $h_{1}^{\prime}$ to $M_{0}$. Let $H_{1}^{\prime}$ be the cycle of $D_{1}$ obtained from $H_{1}$ by replacing $e, f, h_{1}$ by $e^{\prime}, f^{\prime}, h_{1}^{\prime}$. Then $l_{D_{1}}\left(H_{1}^{\prime}\right)=l_{D^{\prime}}\left(H_{1}\right)$ and $C R\left(H_{1}^{\prime}\right) \stackrel{2}{=}$ $C R\left(H_{1}\right)$;

- $D_{2}$ is obtained from $D-\{e, f\}$ by adding arc $h_{2}$. We remind that $h_{2}$ is embedded on the adjusted $B_{0}$ parallel to $R_{1}$. Let $H_{2}^{\prime}=H_{2}$. Then $l_{D_{2}}\left(H_{2}^{\prime}\right)=l_{D^{\prime}}\left(H_{2}\right)$ and $C R\left(H_{2}^{\prime}\right) \stackrel{2}{=} C R\left(H_{2}\right)$.

We remind that $l_{D}(R)=1$. Hence, exactly one of $h_{i}$ is oriented so that both cycles it makes with $R$ are clockwise odd. Let it be $h_{2}$. Then $D_{2}$ is a relevant orientation and $D_{1}$ becomes relevant after reversing the orientation of $h_{1}^{\prime}$ : this digraph, obtained from $D_{1}$ by reversing the orientation of $h_{1}^{\prime}$, we denote by $D_{1}^{*}$, and its subdigraph corresponding to $H_{1}^{\prime}$ we denote by $H_{1}^{*}$. Then, $l_{D_{1}^{*}}\left(H_{1}^{*}\right) \stackrel{2}{=} l_{D_{1}}\left(H_{1}^{\prime}\right)+1$. 
Note that both $D_{2}$ and $D_{1}^{*}$ are relevant orientations of proper $g$-graphs, $H_{2}^{\prime}$ is an alternating cycle of $D_{2}, H_{1}^{*}$ is an alternating cycle of $D_{1}^{*}$ and $C R\left(H_{1}^{*}\right)<C R(C)$ and $C R\left(H_{2}^{\prime}\right)<C R(C)$. Hence, by the induction assumption, we have that:

$$
\begin{aligned}
l_{D}(C) \stackrel{2}{=} & l_{D^{\prime}}\left(H_{1}\right)+l_{D^{\prime}}\left(H_{2}\right) \stackrel{2}{=} l_{D_{1}}\left(H_{1}^{\prime}\right)+l_{D_{2}}\left(H_{2}^{\prime}\right) \stackrel{2}{=} l_{D_{1}^{*}}\left(H_{1}^{*}\right)+1+l_{D_{2}}\left(H_{2}^{\prime}\right) \stackrel{2}{=} \\
& \left|B R\left(H_{1}^{*}\right)\right|-1-C R\left(H_{1}^{*}\right)+\frac{1}{2} \sum_{p \in B R\left(H_{1}^{*}\right)}\left(r\left(D_{1}^{*}\right)_{d(p)}+1\right)+ \\
& \left|B R\left(H_{2}^{\prime}\right)\right|-1-C R\left(H_{2}^{\prime}\right)+\frac{1}{2} \sum_{p \in B R\left(H_{2}^{\prime}\right)}\left(r\left(D_{2}\right)_{d(p)}+1\right)+1 .
\end{aligned}
$$

Now, the theorem follows by observing that $|B R(C)| \stackrel{2}{=}|B R(C-\{e, f\})| \stackrel{2}{=}$ $\left|B R\left(H_{1}^{*}\right)\right|+\left|B R\left(H_{2}^{\prime}\right)\right|-2, C R(C) \stackrel{2}{=} C R\left(H_{1}^{*}\right)+C R\left(H_{2}^{\prime}\right)$ and $r\left(D_{1}^{*}\right)_{d(p)}, r\left(D_{2}\right)_{d(p)}$ and $r(D)_{d(p)}$ coincide for any $p \in B R(C)-\{e, f\}$. Hence,

$$
l_{D}(C) \stackrel{2}{=}|B R(C)|-1-C R(C)+\frac{1}{2} \sum_{p \in B R(C)}\left(r(D)_{d(p)}+1\right) .
$$

(End of Subcase 1.1)

Subcase 1.2: $h_{1}=u_{1} v_{2}$.

Let $h_{1}$ and $h_{2}$ be embedded on the bridge $B$. Observe that $C R(C) \stackrel{2}{=} C R\left(H_{1}\right)+$ $C R\left(H_{2}\right)+1$ : attention again should be drawn to the question of how crossings of $C$ with itself are manifested as crossings of $H_{1}$ or $H_{2}$, when all $E_{j}^{i}$ are projected outside of $B_{0}$ (see Definition 2.3). To see this clearly, we introduce some notation. Let $A$ be a subset of arcs of $H_{1}$ and $B$ a subset of arcs of $H_{2}$. We denote by $C R(A \times B)$ the number of crossings between $\operatorname{arcs}$ of $A$ and $B, \bmod 2$. We also denote by $\operatorname{cr}(i, j)$ the number of crossings of arcs of $H_{i} \cap C$ with $h_{j}$. Hence, we have:

$$
\begin{gathered}
C R\left(H_{1}\right) \stackrel{2}{=} C R\left(H_{1} \cap C\right)+c r(1,1), \\
C R\left(H_{2}\right) \stackrel{2}{=} C R\left(H_{2} \cap C\right)+c r(2,2), \\
C R(C) \stackrel{2}{=} C R\left(H_{1} \cap C\right)+C R\left(H_{2} \cap C\right)+C R\left(\left(H_{1} \cap C\right) \times\left(H_{2} \cap C\right)\right), \\
C R\left(H_{1} \times H_{2}\right) \stackrel{2}{=} 0,
\end{gathered}
$$

and

$$
\sum_{i, j=1}^{2} \operatorname{cr}(i, j) \stackrel{2}{=} 0
$$

since each arc which crosses $h_{1}$ crosses also $h_{2}$.

Hence it remains to show that

$$
C R\left(H_{1} \times H_{2}\right) \stackrel{2}{=} C R\left(\left(H_{1} \cap C\right) \times\left(H_{2} \cap C\right)\right)+\operatorname{cr}(1,2)+\operatorname{cr}(2,1)+1:
$$

this follows since in this case one additional crossing between $H_{1}$ and $H_{2}$ must occur at an endvertex of $h$. The required congruence follows.

We construct two digraphs $D_{1}, D_{2}$ as follows: 
- $D_{1}$ is obtained from $D-\{e, f\}$ by adding a new arc $h_{1}^{\prime}$ between $v_{1}$ and the endvertex $u_{2}$ of $e$. If $l_{D^{\prime}}\left(f h_{1} e\right)=1$ then we let $h_{1}^{\prime}=\left(v_{1}, u_{2}\right)$. If $l_{D^{\prime}}\left(f h_{1} e\right)=0$ then we let $h_{1}^{\prime}=\left(u_{2}, v_{1}\right)$.

We consider $h_{1}^{\prime}$ embedded on the bridge $B$. Let $H_{1}^{\prime}$ be obtained from $H_{1}$ by replacing $\left\{f, h_{1}, e\right\}$ by $h_{1}^{\prime}$. We have $l_{D^{\prime}}\left(H_{1}\right)=l_{D_{1}}\left(H_{1}^{\prime}\right)$ and $C R\left(H_{1}\right)=C R\left(H_{1}^{\prime}\right)$.

- $D_{2}$ is obtained from $D-\{e, f\}$ by adding the arc $h_{2}$. We consider $h_{2}$ embedded on the bridge $B$. We let $H_{2}=H_{2}^{\prime}$. Thus again we have $l_{D^{\prime}}\left(H_{2}\right)=l_{D_{2}}\left(H_{2}^{\prime}\right)$ and $C R\left(H_{2}\right)=C R\left(H_{2}^{\prime}\right)$.

We remind that $l_{D}(R)=1$ and thus exactly one of $h_{i}$ is oriented so that both cycles it makes with $R$ are clockwise odd. Let it be $h_{2}$. Let $R_{3}$ be the subpath of $C_{0}$ from $v_{1}$ to $v_{2}$ such that $\left(e, R_{1}, R_{3}, R_{2}\right)$ is a cycle. We have $l_{D_{1}}\left(h_{1}^{\prime}, R_{3}, R_{2}\right) \stackrel{2}{=}$ $l_{D^{\prime}}\left(e, h_{1}, f, R_{3}, R_{2}\right) \stackrel{2}{=} l_{D^{\prime}}\left(f, R_{3}\right)+l_{D^{\prime}}\left(e, h_{1}, R_{2}\right)$.

We show now that both $D_{1}$ and $D_{2}$ are relevant orientations with $r\left(D_{1}\right)=r\left(D_{2}\right)=$ $r(D)$. We only need to show that $h_{1}^{\prime}$ and $h_{2}$ are correctly oriented in $D_{1}$ and $D_{2}$. This follows easily for $D_{2}$, since both cycles $h_{2}$ makes with $R$ are clockwise odd.

For $D_{1}$ we distinguish two cases. First, let $r(D)_{2(i-1)+j}=1$. In this case we have $l_{D^{\prime}}\left(f, R_{3}\right)=1$ and $l_{D^{\prime}}\left(e, h_{2}, R_{2}\right)=1$. Hence $l_{D^{\prime}}\left(e, h_{1}, R_{2}\right)=0$. It follows that $l_{D_{1}}\left(h_{1}^{\prime}, R_{3}, R_{2}\right)=1$ and $D_{1}$ is relevant with $r\left(D_{1}\right)=r(D)$. Secondly, let $r(D)_{2(i-1)+j}=-1$. In this case we have $l_{D^{\prime}}\left(f, R_{3}\right)=0$ and $l_{D^{\prime}}\left(e, h_{2}, R_{2}\right)=1$. Hence $l_{D^{\prime}}\left(e, h_{1}, R_{2}\right)=0$. It follows that $l_{D_{1}}\left(h_{1}^{\prime}, R_{3}, R_{2}\right)=0$ and $D_{1}$ is relevant with $r\left(D_{1}\right)=r(D)$.

Hence, $D_{i}$ is a relevant orientation of a proper $g$-graph, $H_{i}^{\prime}$ is an alternating cycle of $D_{i}$ and $\left|B R\left(H_{i}^{\prime}\right)\right|<|B R(C)|$, for $i=1,2$, and, by the induction hypothesis, we have that:

$$
\begin{gathered}
l_{D}(C) \stackrel{2}{=} l_{D^{\prime}}\left(H_{1}\right)+l_{D^{\prime}}\left(H_{2}\right) \stackrel{2}{=} l_{D_{1}}\left(H_{1}^{\prime}\right)+l_{D_{2}}\left(H_{2}^{\prime}\right) \stackrel{2}{=} \\
\left|B R\left(H_{1}^{\prime}\right)\right|-1-C R\left(H_{1}^{\prime}\right)+\frac{1}{2} \sum_{p \in B R\left(H_{1}^{\prime}\right)}\left(r\left(D_{1}\right)_{d(p)}+1\right)+\frac{1}{2}\left(r\left(D_{1}\right)_{d\left(h_{1}\right)}+1\right)+ \\
\left|B R\left(H_{2}^{\prime}\right)\right|-1-C R\left(H_{2}^{\prime}\right)+\frac{1}{2} \sum_{p \in B R\left(H_{2}^{\prime}\right)}\left(r\left(D_{2}\right)_{d(p)}+1\right)+\frac{1}{2}\left(r\left(D_{2}\right)_{d\left(h_{2}\right)}+1\right) .
\end{gathered}
$$

The theorem follows by observing that $|B R(C)| \stackrel{2}{=}|B R(C-\{e, f\})| \stackrel{2}{=}\left|B R\left(H_{1}^{\prime}\right)\right|+$ $\left|B R\left(H_{2}^{\prime}\right)\right|-2, C R(C)+1 \stackrel{2}{=} C R\left(H_{1}^{\prime}\right)+C R\left(H_{2}^{\prime}\right)$ and $r\left(D_{1}\right)=r\left(D_{2}\right)=r(D)$.

(End of Subcase 1.2)

End of Case 1

Case 2. There exists $i$ such that $C$ contains exactly one edge from both $E_{1}^{i}$ and $E_{2}^{i}$. Let $e \in E_{1}^{i}$ and $f \in E_{2}^{i}$ and let $C_{1}$ and $C_{2}$ be two paths such that $C=\left(C_{1}, e, C_{2}, f\right)$. The endvertices of $e, f$ belong to $C_{0}$. Let us assume that along the boundary of $B_{0}$ from $a_{4(i-1)+1}$ to $a_{4 i+1}$, the endvertices of $e, f$ appear in the order $v_{1}, u_{1}, v_{2}, u_{2}$ where $e=u_{1} u_{2}$ and $f=v_{1} v_{2}$. 
Let $R_{1}, R_{2}$ be the two disjoint subpaths of the segment of $C_{0}$ between $a_{4(i-1)+1}$ and $a_{4 i+1}$, which cover the endvertices of $e, f$. Note that $R_{1}, R_{2}$ contain no other vertex of $G$ incident with an edge out of $E_{0}$, by the choice of $i$. Let $R$ denote the cycle $\left(R_{1}, e, R_{2}, f\right)$ and let $R_{3}$ denote the segment of $C_{0}$ between $u_{1}$ and $v_{2}$.

Let us introduce a new edge $h$ (not belonging to $G$ ), between endvertices of $e, f$ such that one of two cycles $\bar{I}_{1}, \bar{I}_{2}$ formed by $h$ and $C$ and containing $h$ is alternating.

Without loss of generality let $h$ have $u_{1}$ as an endvertex. Hence we have that $h=u_{1} v_{1}$ or $h=u_{1} v_{2}$. We may also assume without loss of generality that $\bar{I}_{2}$ is alternating. Hence $\bar{I}_{1}$ contains both $e, f$. Note that $\bar{I}_{1}$ consists of an even number of edges.

We denote by $h_{1}, h_{2}$ the two arcs with the same endvertices as $h$, directed oppositely. Let $D^{\prime}=D \cup\left\{h_{1}, h_{2}\right\}$. Let $I_{i}$ be the subdigraph of $D^{\prime}$ which is the orientation of $\bar{I}_{i}$ using $h_{i}, i=1,2$. Observe that $l_{D}(C)=l_{D^{\prime}}\left(I_{1}\right)+l_{D^{\prime}}\left(I_{2}\right)$.

Again we distinguish two subcases.

Subcase 2.1: $h_{1}=u_{1} v_{1}$.

In this case $h$ forms a cycle with $R_{1}$.

As in Subcase 1.1, we extend $B_{0}$ along $R_{1}$ and consider $h_{1}, h_{2}$ as embedded on the extended $B_{0}$.

Observe that $C R(C) \stackrel{2}{=} C R\left(I_{1}\right)+C R\left(I_{2}\right)$ : attention should be drawn to the question of how crossings of $C$ with itself are manifested as crossings of $I_{1}$ or $I_{2}$, when all $E_{j}^{i}$ are projected outside of $B_{0}$ (see Definition 2.3). In this case, the arguments are identical to those used in the proof of Subcase 1.1, and we omit them.

We construct two digraphs $D_{1}, D_{2}$ as follows:

- $D_{1}$ is obtained from $D-\{e, f\}$ by adding new vertices $u_{1}^{\prime}, v_{1}^{\prime}$ of degree 2 , incident with new $\operatorname{arcs} e^{\prime}, f^{\prime}, h_{1}^{\prime}$. The $\operatorname{arcs} e^{\prime} f^{\prime}, h_{1}^{\prime}$ are obtained from $e, f, h_{1}$ by replacing $u_{1}$ by $u_{1}^{\prime}$ and $v_{1}$ by $v_{1}^{\prime}$. We extend $B_{0}$ along $R_{2}$ and we embed the path $\left(e^{\prime}, f^{\prime}, h_{1}^{\prime}\right)$ on the extended $B_{0}$. Finally we add $h_{1}^{\prime}$ to $M_{0}$. Let $I_{1}^{\prime}$ be the cycle of $D_{1}$ obtained from $I_{1}$ by replacing $e, f, g_{1}$ by $e^{\prime}, f^{\prime}, g_{1}^{\prime}$. We have $l_{D^{\prime}}\left(I_{1}\right)=l_{D_{1}}\left(I_{1}^{\prime}\right)$ and $C R\left(I_{1}\right)-1 \stackrel{2}{=} C R\left(I_{1}^{\prime}\right)$.

- $D_{2}$ is obtained from $D-\{e, f\}$ by adding the arc $h_{2}$. We consider $h_{2}$ embedded on the extended $B_{0}$ along $R_{1}$. We let $I_{2}^{\prime}=I_{2}$. Hence, $l_{D^{\prime}}\left(I_{2}\right)=l_{D_{2}}\left(I_{2}^{\prime}\right)$ and $C R\left(I_{2}\right) \stackrel{2}{=} C R\left(I_{2}^{\prime}\right)$.

Hence, for $i=1,2, D_{i}$ is an orientation of a proper $g$-graph and $I_{i}^{\prime}$ is an alternating cycle of $D_{i}$. Moreover $\left|B R\left(I_{i}^{\prime}\right)\right|<|B R(C)|$.

Let us assume without loss of generality that $h_{2}$ is directed so that the cycle $l_{D^{\prime}}\left(R_{1}, h_{2}\right)=1$. Hence $D_{2}$ is a relevant orientation with $r\left(D_{2}\right)=r(D)$.

We show now that $D_{1}$ is a relevant orientation with $r(D)=r\left(D_{1}\right)$ if and only if $r(D)_{d(e)}=r(D)_{d(f)}$. We first prove that if $r(D)_{d(e)}=r(D)_{d(f)}=1$ then $D_{1}$ is a relevant orientation.

In this case it suffices to show that $l_{D_{1}}\left(R_{2}, f^{\prime}, h_{1}^{\prime}, e^{\prime}\right) \stackrel{2}{=} 1$. We have $l_{D_{1}}\left(h_{1}^{\prime}, f^{\prime}, R_{3}\right) \stackrel{2}{=}$ $l_{D^{\prime}}\left(h_{1}, f, R_{3}\right) \stackrel{2}{=} l_{D^{\prime}}\left(h_{2}, f, R_{3}\right)+1 \stackrel{2}{=} l_{D_{2}}\left(h_{2}, f, R_{3}\right)+1 \stackrel{2}{=} 0$, since $r\left(D_{2}\right)_{d(f)}=r(D) d(f)=$ 1 , and thus, $l_{D_{2}}\left(h_{2}, f, R_{3}\right)=1$. 
Moreover $l_{D_{1}}\left(R_{2}, R_{3}, e^{\prime}\right)=l_{D^{\prime}}\left(R_{2}, R_{3}, e\right)=1$, since $r(D)_{d(e)}=1$ and $D$ is a relevant orientation. Replacing $f^{\prime} h_{1}^{\prime}$ for $R_{3}$ gives what we claimed.

Similarly, we can prove that if $r(D)_{d(e)}=r(D)_{d(f)}=-1$ then again $l_{D_{1}}\left(R_{2}, f^{\prime}, h_{1}^{\prime}, e^{\prime}\right)=1$, and so, $D_{1}$ is a relevant orientation.

On the other hand, if $r(D)_{d(e)} \neq r(D)_{d(f)}$ then $D_{1}$ is obtained from a relevant orientation by reversing one arc, and so, it is not relevant.

Summarizing, if $r(D)_{d(e)}=r(D)_{d(f)}$ then $D_{1}$ is a relevant orientation with $r(D)=$ $r\left(D_{1}\right)$, and if $r(D)_{d(e)} \neq r(D)_{d(f)}$ then $D_{1}$ becomes relevant after reversing the orientation of $h_{1}^{\prime}$ : this digraph, obtained from $D_{1}$ by reversing the orientation of $h_{1}^{\prime}$, we denote by $D_{1}^{*}$, and its subdigraph corresponding to $H_{1}^{\prime}$ we denote by $H_{1}^{*}$. Then $l_{D_{1}^{*}}\left(I_{1}^{\prime}\right) \stackrel{2}{=} l_{D_{1}}\left(I_{1}^{\prime}\right)+1$.

Using the induction assumption of 3.1 for $D^{*}, I_{1}^{*}, D_{1}, I_{1}^{\prime}$ and $D_{2}, I_{2}^{\prime}$ we get:

$$
\begin{gathered}
l_{D}(C) \stackrel{2}{=} l_{D^{\prime}}\left(I_{1}\right)+l_{D^{\prime}}\left(I_{2}\right) \stackrel{2}{=} l_{D_{1}}\left(I_{1}^{\prime}\right)+l_{D_{2}}\left(I_{2}^{\prime}\right) \stackrel{2}{=} \\
|B R(C)|-4-C R(C)+1+\frac{1}{2} \sum_{p \in B R(C)-\{e, f\}}\left(r(D)_{d(p)}+1\right)+\frac{1}{2}\left(r(D)_{d(e)}-1+r(D)_{d(f)}-1\right) \stackrel{2}{=} \\
|B R(C)|-1-C R(C)+\frac{1}{2} \sum_{p \in B R(C)}\left(r(D)_{d(p)}+1\right) .
\end{gathered}
$$

(End of Subcase 2.1)

Subcase 2.2: $h=u_{1} v_{2}$.

In this case $h$ forms a cycle with $R_{3}$. We extend $B_{0}$ along $R_{3}$ and consider $h_{1}, h_{2}$ embedded on the extended $B_{0}$.

Observe that $C R(C) \stackrel{2}{=} C R\left(I_{1}\right)+C R\left(I_{2}\right)$ :attention should be drawn to the question of how crossings of $C$ with itself are manifested as crossings of $I_{1}$ or $I_{2}$, when all $E_{j}^{i}$ are projected outside of $B_{0}$ (see Definition 2.3). In this case, the arguments are identical to those used in the proof of Subcase 1.1 and Subcase 2.1, and we omit them.

We construct two digraphs $D_{1}, D_{2}$ as follows:

- $D_{1}$ is obtained from $D-\{e, f\}$ by adding new vertices $u_{1}^{\prime}, v_{2}^{\prime}$ of degree 2 , incident with new $\operatorname{arcs} e^{\prime}, f^{\prime}, h_{1}^{\prime}$. The $\operatorname{arcs} e^{\prime}, f^{\prime}, h_{1}^{\prime}$ are obtained from $e, f, h_{1}$ by replacing $u_{1}$ by $u_{1}^{\prime}$ and $v_{2}$ by $v_{2}^{\prime}$. We extend $B_{0}$ along $R_{1} R_{3} R_{2}$ and we embed $e^{\prime}, f^{\prime}, h_{1}^{\prime}$ on the extended $B_{0}$. Finally we add $h_{1}^{\prime}$ to $M_{0}$. Let $I_{1}^{\prime}$ be the cycle of $D_{1}$ obtained from $I_{1}$ by replacing $e, f, h_{1}$ by $e^{\prime}, f^{\prime}, h_{1}^{\prime}$. We have that $l_{D^{\prime}}\left(I_{1}\right) \stackrel{2}{=} l_{D_{1}}\left(I_{1}^{\prime}\right)$ and $C R\left(I_{1}^{\prime}\right) \stackrel{2}{=} C R\left(I_{1}\right)-1$.

- $D_{2}$ is obtained from $D-\{e, f$,$\} by adding arc h_{2}$. We again extend $B_{0}$ along $R_{3}$ and consider $h_{2}$ embedded on the extended $B_{0}$. We let $I_{2}^{\prime}=I_{2}$. Hence, $l_{D^{\prime}}\left(I_{2}\right) \stackrel{2}{=} l_{D_{2}}\left(I_{2}^{\prime}\right)$ and $C R\left(I_{2}^{\prime}\right) \stackrel{2}{=} C R\left(I_{2}\right)$.

Hence for $i=1,2, D_{i}$ is an orientation of a proper $g$-graph and $I_{i}^{\prime}$ is an alternating cycle of $D_{i}$. Moreover $\left|B R\left(I_{i}^{\prime}\right)\right|<|B R(C)|$.

Let us assume without loss of generality that $h_{2}$ is directed so that $l\left(R_{3}, h_{2}\right)=1$. Hence $D_{2}$ is a relevant orientation with $r\left(D_{2}\right)=r(D)$. 
As in Subcase 2.1, we shall show that $D_{1}$ is a relevant orientation if and only if $r(D)_{d(e)}=r(D)_{d(f)}$ : It again suffices to consider the case that $r(D)_{d(e)}=r(D)_{d(f)}=$ 1. In this case it suffices to show that $l_{D_{1}}\left(R_{2}, R_{3}, R_{1}, f^{\prime}, h_{1}^{\prime}, e^{\prime}\right)=1$. In fact, we have $l_{D_{2}}\left(R_{1}, f, h_{2}\right)=1$ since $r\left(D_{d(f)}\right)=1$ and $D_{2}$ is a relevant orientation. Hence $l_{D_{1}}\left(R_{1}, f^{\prime}, h_{1}^{\prime}\right)=0$. Moreover $l_{D_{1}}\left(R_{2}, R_{3}, e^{\prime}\right) \stackrel{2}{=} l_{D}\left(R_{2}, R_{3}, e\right)=1$ since $r(D)_{d(e)}=1$. Hence $l_{D_{1}}\left(R_{2}, R_{3}, R_{1}, f^{\prime}, h_{1}^{\prime}, e^{\prime}\right)=1$.

Summarizing, if $r(D)_{d(e)}=r(D)_{d(f)}$ then $D_{1}$ is a relevant orientation with $r(D)=$ $r\left(D_{1}\right)$, and if $r(D)_{d(e)} \neq r(D)_{d(f)}$ then $D_{1}$ becomes relevant after reversing the orientation of $h_{1}^{\prime}$.

The proof then proceeds analogously as in Subcase 2.1. (End of Subcase 2.2)

End of Case 2

It is not difficult to see that the two cases complete the proof.

Next we show that a statement analogous to that of Theorem 3.1 holds for the set of the alternating cycles of $M_{0} \Delta P$ as well.

Theorem 3.2 Let $G$ be a proper g-graph and let $D$ be a relevant orientation of $G$. Let $P$ be a perfect matching of $G$. Then

$$
\operatorname{sgn}\left(D, M_{0} \Delta P\right)=(-1)^{q},
$$

where

$$
q \stackrel{2}{=}\left|B R\left(M_{0} \Delta P\right)\right|-C R\left(M_{0} \Delta P\right)+\frac{1}{2} \sum_{e \in B R\left(M_{0} \Delta P\right)}\left(r(D)_{d(e)}+1\right)
$$

Proof. Let $C_{1}, \ldots, C_{k}$ be the alternating cycles of $M_{0} \Delta P$. We have that $\operatorname{sgn}\left(D, M_{0} \Delta P\right)=(-1)^{q}$, where $q \stackrel{2}{=} l\left(C_{1}\right)+\ldots+l\left(C_{k}\right)-k$.

Using Theorem 3.1 for $C_{1}, \ldots, C_{k}$, it remains to show that:

$$
C R\left(M_{0} \Delta P\right) \stackrel{2}{=} \sum_{j=1}^{k} C R\left(C_{j}\right)
$$

but this holds by Lemma 2.8 and the theorem follows.

Corollary 3.3 Let $G$ be a proper 1 -graph and $D$ a relevant orientation of $G$. Let $\mathcal{C}$ be a set of disjoint alternating cycles of $M_{0}$. Then:

1. If $r(D)=(1,1)$ then $\operatorname{sgn}(D, \mathcal{C})=1$ if and only if $t(\mathcal{C}) \in\{(0,0),(0,1),(1,0)\}$.

2. If $r(D)=(1,-1)$ then $\operatorname{sgn}(D, \mathcal{C})=1$ if and only if $t(\mathcal{C}) \in\{(0,0),(1,1),(1,0)\}$.

3. If $r(D)=(-1,1)$ then $\operatorname{sgn}(D, \mathcal{C})=1$ if and only if $t(\mathcal{C}) \in\{(0,0),(0,1),(1,1)\}$.

4. If $r(D)=(-1,-1)$ then $\operatorname{sgn}(D, \mathcal{C})=1$ if and only if $t(\mathcal{C})=(0,0)$. 
Definition 3.4 Let $G$ be a proper $g$-graph and $D$ a relevant orientation of $G$. Let $r(D)=\left(r_{1}, \ldots, r_{2 g}\right)$. We let $c(r(D))$ equal to the product of $c_{i}, i=0, \ldots, g-1$, where $c_{i}=c\left(r_{2 i+1}, r_{2 i+2}\right)$ and $c(1,1)=c(1,-1)=c(-1,1)=1 / 2$ and $c(-1,-1)=-1 / 2$.

Observe that $c(r(D))=(-1)^{n} 2^{-g}$, where $n=\left|\left\{i ; r_{2 i+1}=r_{2 i+2}=-1\right\}\right|$.

Corollary 3.5 Let $G$ be a proper 1 -graph. Let $D_{1}, D_{2}, D_{3}, D_{4}$ be the relevant orientations of $G$. Then

$$
\mathcal{P}(G, x)=\sum_{i=1}^{4} c\left(r\left(D_{i}\right)\right) \mathcal{P}\left(D_{i}, M_{0}\right)
$$

A result analogous to Corollary 3.5 holds for all proper $g$-graphs, $g>1$. In order to deduce it we start with another corollary of Theorem 3.2.

Corollary 3.6 Let $G$ be a proper g-graph and $D$ a relevant orientation of $G$. Let $P$ be a perfect matching of $G$. Then $\operatorname{sgn}\left(D, M_{0} \Delta P\right)$ is a function of $r(D)$ and $t\left(M_{0} \Delta P\right)$ only. Let us denote this function by $\sigma\left(r(D), t\left(M_{0} \Delta P\right)\right)$.

Lemma 3.7 Let $r=\left(r_{1}, \ldots, r_{2 g}\right)$ and $t=\left(t_{1}, \ldots, t_{2 g}\right)$ be $2 g$-dimensional vectors. Let $r(j)=\left(r_{2 j+1}, r_{2 j+2}\right)$ and $t(j)=\left(t_{2 j+1}, t_{2 j+2}\right), j=0, \ldots, g-1$. Then

$$
\sigma(r, t)=\prod_{j=0}^{g-1} \sigma(r(j), t(j)) .
$$

Proof. By Corollary 3.6, we have that $\operatorname{sgn}(D, \mathcal{C})=\operatorname{sgn}\left(D^{\prime}, \mathcal{C}^{\prime}\right)$ if and only if $r(D)=$ $r\left(D^{\prime}\right)$ and $t(\mathcal{C})=t\left(\mathcal{C}^{\prime}\right)$. This implies that we can restrict ourselves to consider the following case: $G=C_{0} \cup M_{0} \cup \mathcal{C}$ is a proper $g$-graph, $D$ is a relevant orientation of $G$ such that $r(D)=r$ and $\mathcal{C}$ consists of a set of vertex-disjoint cycles $C_{1}, \ldots, C_{k}$ satisfying the following properties:

1. each $C_{i}$ is alternating with respect to the perfect matching $M_{0}$,

2. for each $i, j\left|E_{j}^{i}\right| \leq 1$,

3. for each $i$ there is at most one $j$ such that $\left|C_{j} \cap\left(E_{1}^{i} \cup E_{2}^{i}\right)\right| \geq 1$,

4. for each $C_{j}$ there is exactly one $i$ such that $C_{j}$ intersects $E_{1}^{i} \cup E_{2}^{i}$,

5. $t(\mathcal{C})=t$.

Hence,

$$
\sigma(r, t)=\operatorname{sgn}(D, \mathcal{C})=\prod_{i=1}^{k} \operatorname{sgn}\left(D, C_{i}\right)=\prod_{i=1}^{k} \operatorname{sgn}\left(D_{i}, C_{i}\right)
$$

where $D_{i}$ is the restriction of $D$ to $C_{0} \cup C_{i}$. Observe that, by Corollary 3.3, $\sigma\left(z_{1}, z_{2}\right)=1$ if $z_{2}=(0,0)$. Hence, using Corollary 3.6, we have that $\prod_{i=1}^{k} \operatorname{sgn}\left(D_{i}, C_{i}\right)=$ $\prod_{j=0}^{g-1} \sigma\left(r^{j}, t^{j}\right)$ as claimed. 
Theorem 3.8 Let $G$ be a proper g-graph. Then

$$
\mathcal{P}(G, x)=\mathcal{L}_{g}(G, x)=\sum_{i=1}^{4^{g}} c\left(r\left(D_{i}\right)\right) \mathcal{P}\left(D_{i}, M_{0}\right)
$$

where $D_{i}, i=1, \ldots, 4^{g}$, are the relevant orientations of $G$.

Proof. Let $P$ be a perfect matching of $G$. In each term $\mathcal{P}\left(D_{i}, M_{0}\right)$, the coefficient of $x(P)$ is $\operatorname{sgn}\left(D_{i}, M_{0} \Delta P\right)$. By Corollary 3.6, $\operatorname{sgn}\left(D_{i}, M_{0} \Delta P\right)=\sigma\left(r\left(D_{i}\right), t\left(M_{0} \Delta P\right)\right)$.

Let

$$
\mathcal{K}_{g}\left(t\left(M_{0} \Delta P\right)\right)=\sum_{i=1}^{4^{g}} c\left(r\left(D_{i}\right)\right) \sigma\left(r\left(D_{i}\right), t\left(M_{0} \Delta P\right)\right)
$$

denote the coefficient of $x(P)$ in $\mathcal{L}_{g}(G, x)$.

To prove the theorem it suffices to prove the following claim:

Claim. $\mathcal{K}_{g}\left(t\left(M_{0} \Delta P\right)\right)=1$ for each $t\left(M_{0} \Delta P\right)$.

The proof of the claim is by induction on $g$. The basis of the induction when $g=1$ is proved in Corollary 3.5.

To prove the inductive step we introduce the following notation: if $z$ is a $2 g$ dimensional vector then we let $z=(z(0), \ldots, z(g-1))$ where $z(i)=\left(z_{2 i+1}, z_{2 i+2}\right)$.

We call two relevant orientations $D$ and $D^{\prime}$ of $G$ equivalent if $(r(D)(1), \ldots, r(D)(g-$ $1))=\left(r\left(D^{\prime}\right)(1), \ldots, r\left(D^{\prime}\right)(g-1)\right)$. Clearly, the equivalence classes consist of 4 elements; let $\mathcal{R}_{1}, \ldots, \mathcal{R}_{4^{g-1}}$ be the equivalence classes of the relevant orientations of $G$ and let $\mathcal{R}_{j}=\left\{D_{1}^{j}, D_{2}^{j}, D_{3}^{j}, D_{4}^{j}\right\}, j=1, \ldots, 4^{g-1}$.

Finally let $r\left(D_{i}^{j}\right)(k)=r_{i}^{j}(k), k=0, \ldots, g-1$ and let $t=t\left(M_{0} \Delta P\right)$. We have that

$$
\mathcal{K}_{g}(t)=\sum_{j=1}^{4^{g-1}} \sum_{i=1}^{4} c\left(r\left(D_{i}^{j}\right)\right) \sigma\left(r\left(D_{i}^{j}\right), t\right)
$$

Now, by Lemma 3.7, this equals

$$
\sum_{j=1}^{4^{g-1}} \sum_{i=1}^{4} c\left(r_{i}^{j}(0)\right) c\left(r_{i}^{j}(1), \ldots, r_{i}^{j}(g-1)\right) \prod_{k=0}^{g-1} \sigma\left(r_{i}^{j}(k), t(k)\right) .
$$

By the definition of the equivalence classes, $r_{1}^{j}(k)=r_{2}^{j}(k)=r_{3}^{j}(k)=r_{4}^{j}(k)$ for $k \geq 1$ and $j=1, \ldots, 4^{g-1}$. Hence, we let $r_{i}^{j}(k)=r^{j}(k)$ and write the above summation as:

$$
\sum_{j=1}^{4^{g-1}} c\left(r^{j}(1), \ldots, r^{j}(g-1)\right) \prod_{k=1}^{g-1} \sigma\left(r^{j}(k), t(k)\right) \sum_{i=1}^{4} c\left(r_{i}^{j}(0)\right) \sigma\left(r_{i}^{j}(0), t(0)\right)
$$

The internal sum equals to 1 for each $j=1, \ldots, 4^{g-1}$ by the basis step of the induction, and hence, using Lemma 3.7 in the external sum, we can write the above summation as 


$$
\begin{gathered}
\sum_{j=1}^{4^{g-1}} c\left(r^{j}(1), \ldots, r^{j}(g-1)\right) \sigma\left(\left(r^{j}(1), \ldots, r^{j}(g-1)\right),(t(1), \ldots, t(g-1))\right)= \\
\mathcal{K}_{g-1}(t(1), \ldots, t(g-1))=1,
\end{gathered}
$$

by the inductive hypothesis for $g-1$.

End of Claim.

As a consequence of Theorem 1.4 and Theorem 3.8, we have:

Corollary 3.9 Let $G$ be a proper g-graph. Then $s\left(D_{i}, M_{0}\right)=s\left(D_{j}, M_{0}\right)$ for each $i, j \in\left\{1, \ldots, 4^{g}\right\}$ and

$$
\mathcal{P}(G, x)=\mathcal{L}_{g}(G, x)=s\left(D_{1}, M_{0}\right) \sum_{i=1}^{4^{g}} c\left(r\left(D_{i}\right)\right) \operatorname{Pf}\left(A\left(D_{i}\right)\right)
$$

where $D_{i}, i=1, \ldots, 4^{g}$, are the relevant orientations of $G$.

Theorem 3.10 Let $G$ be a graph embeddable on an orientable surface of genus $g$. Then $\mathcal{P}(G, x)$ may be expressed as a linear combination of $4^{g}$ Pfaffians of matrices $A(D)$, where each $D$ is an orientation of $G$.

Proof. As observed in the previous section, any orientable surface $\mathcal{S}$ of genus $g$ may be obtained from its polygonal representation $S_{g}$ as follows: for each bridge $B$, glue together the two segments in which $B$ intersects the boundary of $B_{0}$, and delete $B$.

If a graph $G$ is embedded on an orientable surface $\mathcal{S}$ of genus $g$, then without loss of generality no vertex belongs to the boundary of $B_{0}$. In this way we get an embedding of $G$ on $S_{g}$ such that all vertices of $G$ belong to $B_{0}$ but the embeddings of some edges may use several bridges.

We construct a graph $G^{\prime}$ by replacing each edge $e=u v$ which uses $k$ bridges, $k \geq 1$, by a path $P_{e}=\left(u, e_{1}, v_{1}, \ldots, v_{2 k}, e_{2 k+1}, v\right)$. The new vertices $v_{1}, \ldots, v_{2 k}$ are embedded on the embedding of $e$ so that each new edge uses at most one bridge. Moreover, we let $x_{e_{1}}^{\prime}=x_{e}$ and $x_{e_{i}}^{\prime}=1$ for each $i>1$. We do a similar construction when $G_{0}$ has no perfect matching. In fact, take any perfect matching $M$ of $G$ and replace any edge $e=u v \in M$ embedded on a bridge by a path $u, e_{1}, y, e_{2}, z, e_{3}, v$ and let $x_{e_{1}}^{\prime}=x_{e}$ and $x_{e_{2}}^{\prime}=x_{e_{3}}^{\prime}=1$. Then leave the only edge $e_{2}$ to be embedded on the bridge $B$.

Finally, we add edges so that the outer face of the planar part is a cycle and we let $x_{e}^{\prime}=0$ for each such edge $e$.

It is easy to see that $G^{\prime}$ is a proper $g$-graph and that $\mathcal{P}\left(G^{\prime}, x^{\prime}\right)=\mathcal{P}(G, x)$.

Now, by Theorem $3.8, \mathcal{P}\left(G^{\prime}, x^{\prime}\right)$ may be written as a linear combination of $4^{g}$ terms $\operatorname{Pf}\left(A\left(D^{\prime}\right)\right)$, where each $D^{\prime}$ is a relevant orientation of $G^{\prime}$.

It remains to show that for each relevant orientation $D^{\prime}$ of $G^{\prime}$ there is an orientation $D$ of $G$ such that $\operatorname{Pf}\left(A\left(D^{\prime}\right)\right)=\operatorname{Pf}(A(D))$ or $\operatorname{Pf}\left(A\left(D^{\prime}\right)\right)=-\operatorname{Pf}(A(D))$.

We construct $D$ from $D^{\prime}$ in two steps: 
1. delete the edges $e$ of $G^{\prime}-G$ with $x_{e}^{\prime}=0$,

2. for each edge $e$ of $G$ which was changed into a path $P_{e}$ of odd length in the construction of $G^{\prime}$, orient $e$ in the direction in which an odd number of edges of $P_{e}$ is directed in $D^{\prime}$ : this is uniquely determined since $P_{e}$ has an odd length.

If $P$ is a perfect matching of $G$ then there is a unique perfect matching $P^{\prime}$ of $G^{\prime}$ such that $x(P)=x^{\prime}\left(P^{\prime}\right)$.

Observe that $\operatorname{sgn}(D, P \Delta Q)=\operatorname{sgn}\left(D^{\prime}, P^{\prime} \Delta Q^{\prime}\right)$ for each pair of perfect matchings $P, Q$ of $G$. The claim now follows from Theorem 1.4.

This finishes the proof of the theorem.

\section{Pfaffian Graphs, Exact Matching, and Perma- nents}

The results of the previous section have interesting algorithmic implications.

Theorem 4.1 Let $g$ and $k$ be fixed positive integers. Let $\mathcal{G}$ be the class of graphs of genus $g$ whose edges are partitioned into at most $k$ classes and the variables $x_{e}$ have the same value in each class. Then $\mathcal{P}(G, x)$ may be determined in polynomial time for $G \in \mathcal{G}$.

Proof. It follows from Theorems 3.8 and 3.10 that $\mathcal{P}(G, x)$ may be expressed as a linear combination of a finite number of Pfaffians.

We show now that if the set of the edges of graph $G$ is partitioned into a bounded number of classes and the variables $x_{e}$ are equal in each class, then $\mathcal{P}(D, M)$ and $\operatorname{Pf}(A(D))$ may be determined efficiently. Let $M=\left\{\left\{i_{1} j_{1}\right\}, \ldots,\left\{i_{n} j_{n}\right\}\right\}, i_{k}<j_{k}$, be a perfect matching of $G$. Let $x^{\prime}$ be defined as follows: $x_{e}^{\prime}=x_{e}$ if $e \notin M$ and $x_{f}^{\prime}=x_{f} z$ if $f \in M$, where $z$ is a new variable. Let $A^{\prime}$ be the matrix obtained from $A(D)$ by replacing each $x_{e}$ by $x_{e}^{\prime}$. Then $\operatorname{det}\left(A^{\prime}\right)$ may be viewed as a polynomial $\operatorname{det}\left(A^{\prime}\right)(x, z)$ in the variables $x$ and $z$ and its coefficients can be determined efficiently.

By Theorem 1.5, $\operatorname{Pf}\left(A^{\prime}\right)(x, z)= \pm \sqrt{\operatorname{det}\left(A^{\prime}\right)(x, z)}$. Hence we can determine efficiently a polynomial $Q(x, z)$ such that $\left.\operatorname{Pf}\left(A^{\prime}\right)(x, z)= \pm Q(x, z)\right)$. Note that $\mathcal{P}(D, M)= \pm Q(x, 1)$.

There is exactly one monomial in $Q(x, z)$ containing $z^{n}$ and its coefficient is +1 or -1 . Let $Q^{\prime}(x, z)$ be the unique polynomial such that $Q^{\prime}(x, z)=Q(x, z)$ or $Q^{\prime}(x, z)=$ $-Q(x, z)$ and the coefficient of $Q^{\prime}(x, z)$ of the term containing $z^{n}$ equals +1 . We have $\mathcal{P}(D, M)=+Q^{\prime}(x, 1)$. Moreover, $\operatorname{Pf}(A(D))=s(D, M) \mathcal{P}(D, M)$ and $s(D, M)=$ $s^{*}(M) t^{*}(M)$ where $t^{*}(M)$ equals the product of the signs of the elements $a_{i_{k} j_{k}}$ of the matrix $A(D)$ such that $i_{k} j_{k} \in M$. Hence $\mathcal{P}(D, M)$ and $P f(A(D))$ may be determined efficiently.

As a consequence, if we are in the hypothesis stated by the theorem, the following problems may be solved efficiently. 
1. Recognition of Pfaffian graphs: given a graph $G \in \mathcal{G}$, decide whether $G$ admits a Pfaffian orientation.

It was proved by Vazirani and Yannakakis (see the proof of Theorem 3.1 in [13]) that, given a graph $G$, it is possible to construct efficiently an orientation $D$ of $G$ such that $G$ is Pfaffian if and only if $D$ is its Pfaffian orientation. Hence $\operatorname{Pf}(A(D))$ equals to the number of the perfect matchings of $G$ if and only if $G$ is Pfaffian, and it means that we can decide efficiently whether a graph is Pfaffian once we can compute efficiently its number of perfect matchings.

2. Exact Matching Problem: given a graph $G \in \mathcal{G}$ with some edges coloured red, and a number $h$, decide whether $G$ has a perfect matching with exactly $h$ red edges.

It suffices to assign an $x$ variable to red edges and a $y$ variable to the remaining edges and compute $\mathcal{P}(G ; x, y)$. If the coefficient of the monomial $x^{h} y^{t-h}$, where $t$ is the cardinality of a perfect matching of $G$, is nonzero then the answer to the problem is yes, otherwise no such a matching exists.

\section{Computing permanents of square matrices.}

In 1913, Pólya [9] suggested computing the permanent of a matrix $A$ by changing the signs of some entries of $A$ so that the determinant of the resulting matrix equals the permanent of $A$. Let us call a $(0,1)$-matrix $A$ convertible if such a change is possible.

Szegö [11] pointed out in the same year that not all matrices are convertible.

This may be explained nowadays using a complexity argument. There is an efficient algorithm to compute the determinant, while Valiant proved that the problem of computing the permanent of a $(0,1)$-matrix is \#P-complete [12].

The computational problem of recognition of convertible matrices has been proved recently to admit a polynomial algorithm by McCuaig, Robertson, Seymour and Thomas [8]. An earlier paper of Galluccio and Loebl [3] contains a related algorithmic result, as well as a history of the problem.

The problem of recognizing convertible matrices is equivalent to the problem of recognizing bipartite Pfaffian graphs, and to the Even Cycle Problem: given a directed graph, decide whether it contains a directed cycle of even length.

Let $A$ be a square matrix. Denote by $G(A)$ the bipartite graph whose two bipartition classes are indexed by the rows and the columns of $A$, and for each edge $i j$, $a_{i j}=x_{i j}$. Then $\operatorname{per}(A)=\mathcal{P}(G(A), x)$.

Hence, Theorem 3.10 provides a new combinatorial way to compute permanents of square matrices: $\operatorname{per}(A)$ may be written as a linear combination of $4^{g}$ terms of form $\operatorname{Pf}(A(D))$, where $D$ is an orientation of $G(A)$ and $g$ is the genus of $G(A)$.

Since $G(A)$ is a bipartite graph, the non-zero entries of $A(D)$ belong to two blocks $A_{1}, A_{2}$, where $A_{1}$ is obtained from $A$ by changing the sign of some entries and $A_{2}=-A_{1}$. Moreover $\mid \operatorname{Pf}\left(A(D)|=| \operatorname{det}\left(A_{1}\right)|=| \operatorname{det}\left(A_{2}\right) \mid\right.$ by Theorem 1.5.

This means that the method of Pólya may be completed as follows:

Corollary 4.2 Let $A$ be a square matrix. Then per $(A)$ may be expressed as a linear combination of terms of the form $\operatorname{det}\left(A^{i}\right), i=1, \ldots, 4^{g}$, where each $A^{i}$ is obtained from $A$ by changing the sign of some entries. 
The ElECtronic Journal of COMBinatorics 6 (1999), \#R6

Acknowledgements. The authors are grateful to Chris Godsil, Robin Thomas and Dominic Welsh for fruitful discussions, and to the referee for the comments which highly improved the quality of the paper.

\section{References}

[1] F. Barahona. Balancing signed toroidal graphs in polynomial time. Preprint University of Chile, 1983. Unpublished manuscript.

[2] A. Cayley. Sur les determinants gauches. Crelle's J., 38:93-96, 1847.

[3] A. Galluccio and M. Loebl. Even cycles and H-homeomorphisms. Technical Report 424, IASI-CNR, 1995. Submitted for publication.

[4] M. Kac and J.C. Ward. A combinatorial solution of the two-dimensional Ising model. Physical Review, 88, 1952.

[5] P. W. Kasteleyn. The statistics of dimers on a lattice. Physica, 27:1209-1225, 1961.

[6] P. W. Kasteleyn. Dimer statistics and phase transitions. Jour. Math. Physics, 4:287-293, 1963.

[7] P.W. Kasteleyn. Graph theory and crystal physics. In Graph theory and theoretical physics, New York, 1967. Academic Press.

[8] W. McCuaig, N. Robertson, P. D. Seymour, and R. Thomas. Permanents, pfaffian orientations, and even directed circuits. 1996. Preprint.

[9] G. Pólya. Aufgabe 424. Arch. Math. Phys., 20:271, 1913.

[10] R.B. Potts and J.C. Ward. The combinatorial method and the two-dimensional Ising model. Progress of Theoretical Physics, 13, 1955.

[11] G. Szegö. Lozung zu 424. Arch. Math. Phys., 21:291-292, 1913.

[12] L. G. Valiant. The complexity of computing the permanent. Theoret. Comput. Sci., 8:189-201, 1979.

[13] V. V. Vazirani and M. Yannakakis. Pfaffian orientations, 0-1 permanents and even cycles in directed graphs. Discr. Appl. Math., 25:179-190, 1989. 